\title{
GABRIELA RIBEIRO VIOLA FERREIRA
}

\section{Avaliação da reserva ovariana e anticorpo anti-corpo}

lúteo em pacientes adolescentes e adultas jovens com artrite idiopática juvenil

\author{
Tese apresentada à Faculdade de Medicina da \\ Universidade de São Paulo para obtenção do título de \\ Doutor em Ciências \\ Programa de Pediatria \\ Orientador: Prof. Dr. Clovis Artur Almeida da Silva
}

São Paulo 


\title{
GABRIELA RIBEIRO VIOLA FERREIRA
}

\section{Avaliação da reserva ovariana e anticorpo anti-corpo}

lúteo em pacientes adolescentes e adultas jovens com artrite idiopática juvenil

\author{
Tese apresentada à Faculdade de Medicina da \\ Universidade de São Paulo para obtenção do título de \\ Doutor em Ciências \\ Programa de Pediatria \\ Orientador: Prof. Dr. Clovis Artur Almeida da Silva
}

São Paulo 


\section{Dados Internacionais de Catalogação na Publicação (CIP)}

Preparada pela Biblioteca da

Faculdade de Medicina da Universidade de Săo Paulo

Creprodução autorizada pelo autor

Ferreira, Gabriela Ribeiro Viola

Avaliação da reserva ovariana e anticorpo anti-

corpo lúteo em pacientes adolescentes e adultas

jovens com artrite idiopática juvenil / Gabriela

Ribeiro Viola Ferreira. -- São Paulo, 2020.

Tese (doutorado) --Faculdade de Medicina da

Universidade de São Paulo.

Programa de Pediatria.

Orientador: Clovis Artur Almeida da Silva.

Descritores: 1.Artrite idiopática juvenil

2.Reserva ovariana 3.Hormônio anti-Mülleriano

4.Fertilidade 5.Anticorpo anti-corpo lúteo

6. Menarca

$\mathrm{USP} / \mathrm{FM} / \mathrm{DBD}-160 / 20$

Responsável: Erinalva da Conceiçăo Batista, CRB-8 6755 


\section{DEDICATÓRIA}

Aos meus pais, Eduardo e Isabel, que sempre me apoiaram e sonharam comigo os meus sonhos.

Ao meu amado esposo Antonio, meu melhor amigo e companheiro incansável nesta trajetória.

À minha querida Helena, luz da minha vida. 


\section{AGRADECIMENTOS}

Primeiramente, agradeço a Deus que me deu a vida e a renova a cada dia, ofertando-me carinhosamente oportunidades pessoais e profissionais.

À minha família, minha essência, obrigada pelo incentivo diário e porto seguro!

Ao meu querido pai, Eduardo, por ser tão presente e não medir esforços para auxiliar a minha jornada.

À minha amada mãe, Isabel, por ser minha grande amiga e abraço reconfortante a qualquer hora. Não tenho palavras para agradecer tudo o que faz por mim.

Ao meu esposo, Antonio, pelo apoio essencial em cada detalhe, pelo carinho nas atitudes mais simples, por caminhar ao meu lado e entender os momentos de ausência.

À minha filhinha amada, Helena, por ser a filha que eu sempre sonhei: carinhosa, companheira e cheia de vida! Motiva-me a ser uma pessoa melhor.

À minha querida irmã, Marcela, amiga de uma vida inteira, minha outra metade. Suas palavras de motivação e alegria sempre me impulsionam a ir além. Obrigada!

À minha querida sogra, Maria de Jesus, pelo apoio diário, principalmente durante todo o período da realização desta tese e pelo auxílio com a correção ortográfica.

Ao meu amado orientador, Prof. Dr. Clovis Artur Almeida da Silva, um grande incentivador de pessoas! Obrigada por me acolher tão calorosamente na família "reumatologia pediátrica do Instituto da Criança e do Adolescente da 
FMUSP" e, principalmente, por me ensinar o valor do estudo e do conhecimento na jornada de um médico. Sem o seu incentivo diário e entusiasmo contagiante, minha trajetória pessoal e profissional não seria a mesma. Agradeço por todos os ensinamentos ao longo desses anos.

Agradeço também às queridas assistentes da "família reumato" que tanto contribuíram para o meu crescimento. À Dra. Lúcia Campos, Dra. Adriana Maluf, Dra. Nádia Aikawa e Dra. Kátia Kozu agradeço imensamente pelo acolhimento e pela convivência tão harmoniosa, pelo carinho no dia-a-dia, pelas discussões de casos no ambulatório e pelos exemplos diários de empatia e humanização. Sou eternamente grata por ter tido o privilégio de conviver e aprender com vocês.

À Dra. Elizabeth Canova e Dra. Ana Paola Lotito que também são de grande importância em minha formação em reumatologia pediátrica, minha gratidão.

Ao meu grande parceiro nesse projeto, Renato Tomioka, excelente médico e auxílio fundamental para que esta pesquisa acontecesse. Obrigada pela dedicação, desde o início!

À Vilma Viana e Elaine Leon do Laboratório de Imunologia Humoral da Disciplina de Reumatologia da FMUSP pelo auxílio com as análises laboratoriais e aos funcionários do ambulatório da Disciplina de Ginecologia da FMUSP, meu agradecimento.

Aos companheiros de especialização, Izabel Buscatti, Camila França, Maria Fernanda Giacomin, Roberta Gomes, Victor Marques e, em especial, a querida e fiel companheira Daniela Lourenço, minha gratidão. Compartilharam comigo os momentos de formação na Unidade de Reumatologia Pediátrica do Instituto da Criança e do Adolescente e se tornaram grandes amigos. Também 
sou grata a todos os pós-graduandos e residentes com quem, ao longo desses anos, eu tive o prazer de conviver e compartilhar experiências.

À grande amiga Natali Gormezano que foi mais um lindo presente que a reumatologia me deu! Gratidão por sua companhia e amizade.

Ao Dr. Ulysses Dória Filho que me auxiliou nas análises estatísticas, sempre com disposição e paciência para ensinar, o meu agradecimento.

Também sou grata ao Nivaldo Rocha que desde a época da especialização em reumatologia foi um grande auxílio no dia-a-dia e agora com a finalização desta tese.

À querida Mariza Kazue, pela generosidade e apoio constante, com grande contribuição na pesquisa bibliográfica e à Mônica Souza por toda ajuda prestada durante o período da pós-graduação.

E não poderia deixar de agradecer aos queridos pacientes que têm iluminado esta minha estrada do conhecimento com carinho e compreensão. 
"Dê-me, Senhor, agudeza para entender, capacidade para reter, método e faculdade para aprender, sutileza para interpretar, graça e abundância para falar. Dême, Senhor, acerto ao começar, direção ao progredir e perfeição ao concluir." Santo Tomás de Aquino, OP 
Esta tese está de acordo com as seguintes normas, em vigor no momento desta publicação:

Referências: adaptado de International Committee of Medical Journals Editors (Vancouver)

Universidade de São Paulo. Faculdade de Medicina. Divisão de Biblioteca e Documentação. Guia de apresentação de dissertações, teses e monografias. Elaborado por Annelise Carneiro da Cunha, Maria Julia de A.L. Freddi, Maria F. Crestana, Marinalva de Souza Aragão, Suely Campos Cardoso, Valéria Vilhena. $3^{a}$ ed. São Paulo. Divisão de Biblioteca e Documentação: 2011.

Abreviaturas dos títulos dos periódicos de acordo com List of Journals Indexed in Index Medicus. 


\section{SUMÁRIO}

LISTA DE ABREVIATURAS E SIGLAS

LISTA DE TABELAS

RESUMO

ABSTRACT

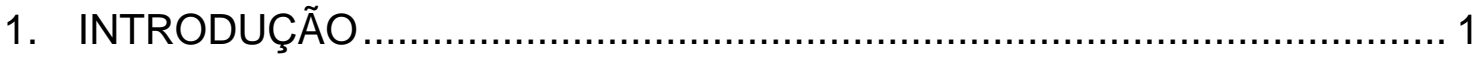

2. OBJETIVOS

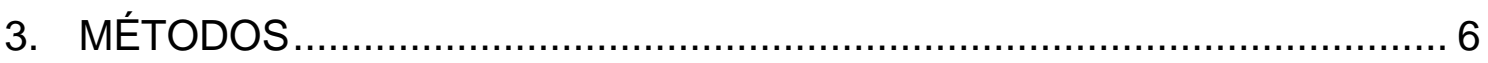

3.1. Desenho do estudo e população ..................................................... 6

3.2. Dados demográficos ........................................................................ 7

3.3. Avaliação ginecológica ....................................................................

3.4. Parâmetros da reserva ovariana ………………............................. 8

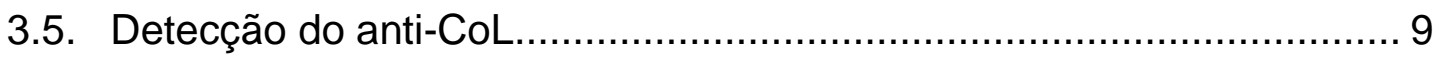

3.6. Parâmetros da doença, qualidade de vida relacionada à saúde e

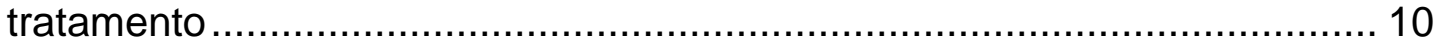

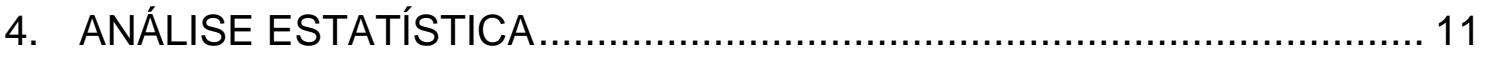

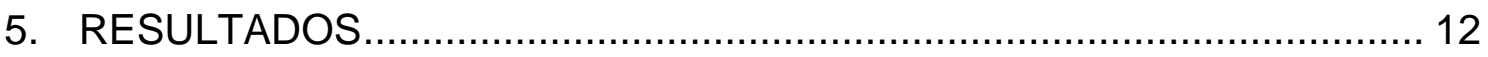

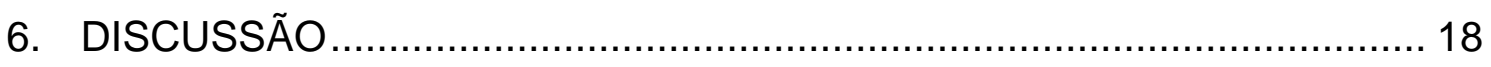

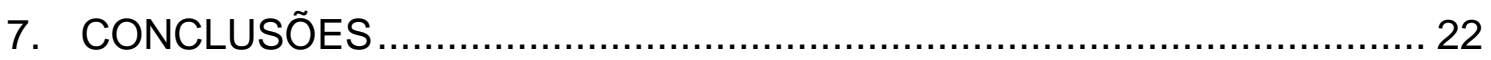

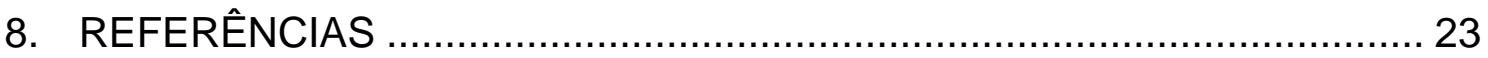




\section{LISTA DE ABREVIATURAS E SIGLAS}

AlJ Artrite idiopática juvenil

Anti-CoL Anticorpo anti-corpo lúteo

BCIP Fosfato 5-bromo-4-cloro-3-indolil

CFA Contagem de folículos antrais

CHAQ Childhood Health Assessment Questionnaire

DAS 28 Disease Activity Score 28-Joint Counts

DMARDs Drogas antirreumáticas modificadoras de doença

DMJ Dermatomiosite juvenil

DP Desvio padrão

EVA Escala visual analógica

FSH Hormônio folículo estimulante

HAM Hormônio anti-Mülleriano

HAQ Health Assessment Questionnaire

HC-FMUSP Hospital das Clínicas da Faculdade de Medicina da Universidade de São Paulo

ILAR

Liga Internacional das Associações de Reumatologia

IMC

Índice de massa corporal

JADAS $71 \quad J u v e n i l e$ Arthritis Disease Activity Score

LESJ Lúpus eritematoso sistêmico juvenil

LH Hormônio luteinizante

MTX Metotrexato

PBS Tampão fosfato salino

PCR Proteína C-reativa 
PedsQL

ROD

SDS-PAGE

SF 36

VHS

Pediatric Quality of Life Inventory

Reserva ovariana diminuída

Eletroforese em gel de poliacrilamida

Short-form 36-item questionnaire

Velocidade de hemossedimentação 


\section{LISTA DE TABELAS}

Tabela 1 - Dados demográficos e avaliação ginecológica em pacientes com artrite idiopática juvenil (AIJ) e controles saudáveis 13

Tabela 2 - Parâmetros hormonais, parâmetros ovarianos e anticorpo anti- -corpo lúteo em pacientes com artrite idiopática juvenil (AIJ) e controles saudáveis 14

Tabela 3 - Avaliação hormonal em pacientes com artrite idiopática juvenil (AIJ) que utilizaram metotrexato (MTX) acima da dose cumulativa de 5 gramas versus aqueles que usaram dose cumulativa abaixo de 5 gramas 15

Tabela 4 - Avaliação hormonal em pacientes com artrite idiopática juvenil (AIJ) que utilizaram ou não agentes biológicos. 16

Tabela 5 - Avaliação hormonal em pacientes com artrite idiopática juvenil (AIJ) cujo início da AlJ ocorreu após a menarca versus início da AlJ antes da menarca 17 


\section{RESUMO}

Ferreira GRV. Avaliação da reserva ovariana e anticorpo anti-corpo lúteo em pacientes adolescentes e adultas jovens com artrite idiopática juvenil [tese]. São Paulo: Faculdade de Medicina, Universidade de São Paulo; 2020.

Objetivos: A artrite idiopática juvenil (AIJ) ocorre durante a idade reprodutiva, no entanto, não existem dados sistematizados sobre a função ovariana nessa doença. Métodos: Vinte e oito pacientes com AlJ pós-púberes e 28 controles saudáveis pareados por idade foram estudados. A função ovariana completa foi avaliada durante a fase folicular precoce do ciclo menstrual, incluindo hormônio anti-Mülleriano (HAM), estradiol, hormônio luteinizante ( $\mathrm{LH}$ ), hormônio folículo estimulante (FSH), contagem de folículos antrais (CFA) por ultrassom ovariano e anticorpo anti-corpo lúteo (anti-CoL). Dados demográficos, anormalidades menstruais, parâmetros da doença e tratamento também foram avaliados. Resultados: A média da idade atual (22,6 $\pm 6,59$ vs. 22,5 $\pm 6,59$ anos, $p=0,952)$ foi semelhante em pacientes com AIJ e controles saudáveis, com mediana da idade da menarca significantemente maior em pacientes com AlJ [13 (8-16) vs. 12 (8-14) anos, $p=0,029]$. Mediana menor em relação aos níveis de HAM [2,65 $(0,47-9,08)$ vs. $4,83(0,74-17,24) \mathrm{ng} / \mathrm{mL}, \mathrm{p}=0,029]$ com maiores níveis de $\mathrm{LH}$ [8,44 $\pm 4,14$ vs. $6,03 \pm 2,80 \mathrm{UI} / \mathrm{L}, \mathrm{p}=0,014]$ e de estradiol foram observados $[52,3(25,8-227,4)$ vs. $38,9(26,2-133,6) \mathrm{pg} / \mathrm{mL}, \mathrm{p}=0,008]$ nas pacientes com AlJ comparadas ao grupo controle. Anti-CoL e CFA foram semelhantes nos dois grupos $(p>0,05)$. Uma análise mais detalhada dos pacientes com AlJ revelou que idade atual, duração da doença, número de articulações ativas/limitadas, velocidade de hemossedimentação (VHS), proteína C-reativa (PCR), escala visual analógica (EVA) paciente/médico, Juvenile Arthritis Disease Activity Score (JADAS 71), Disease Activity Score 28-Joint Counts (DAS 28), Childhood Health Assessment Questionnaire (CHAQ), Health Assessment Questionnaire (HAQ), Pediatric Quality of Life Inventory (PedsQL) paciente/pais, função física do Short-form 36-item questionnaire (SF 36), doses cumulativas de corticosteroide e metotrexato não foram correlacionadas com níveis de HAM, FSH, estradiol ou CFA ( $p>0,05)$. Conclusões: O presente estudo foi o primeiro a sugerir diminuição da reserva ovariana, não associada ao eixo hipotálamo-hipófisegônada, em pacientes com AlJ em idade reprodutiva. $O$ impacto dessa disfunção na fertilidade futura dessas pacientes precisa ser avaliado em estudos prospectivos.

Descritores: Artrite idiopática juvenil; Reserva ovariana; Hormônio antiMülleriano; Fertilidade; Anticorpo anti-corpo lúteo; Menarca. 


\section{ABSTRACT}

Ferreira GRV. Evaluation of ovarian reserve and anti-corpus luteum antibody in adolescent and young adult patients with juvenile idiopathic arthritis [thesis]. São Paulo: "Faculdade de Medicina, Universidade de São Paulo"; 2020.

Objectives: Juvenile idiopathic arthritis (JIA) occurs during reproductive age, however, there are not systematic data regarding ovarian function in this disease. Methods: Twenty-eight post-pubertal JIA patients and age-matched 28 healthy controls were studied. Complete ovarian function was assessed during the early follicular phase of the menstrual cycle including anti-Müllerian hormone $(A M H)$, estradiol, luteinizing hormone ( $\mathrm{LH})$, follicle stimulating hormone (FSH) and antral follicle count (AFC) by ovarian ultrasound, and anti-corpus luteum antibodies (anti-CoL). Demographic data, menstrual abnormalities, disease parameters and treatment were also evaluated. Results: The mean current age (22.6 \pm 6.59 vs. $22.5 \pm 6.59$ years, $p=0.952)$ was similar in JIA patients and healthy controls with a higher median menarche age [13 (8-16) vs. 12 (8-14) years, $p=0.029]$. A lower median AMH levels [2.65 (0.47-9.08) vs. $4.83(0.74-17.24) \mathrm{ng} / \mathrm{mL}, \mathrm{p}=0.029]$ with a higher $\mathrm{LH}[8.44 \pm 4.14$ vs. $6.03 \pm 2.80 \mathrm{IU} / \mathrm{L}, \mathrm{p}=0.014]$ and estradiol levels [52.3 (25.8-227.4) vs. 38.9 (26.2-133.6) $\mathrm{pg} / \mathrm{mL}, \mathrm{p}=0.008$ ] were observed in JIA compared to control group. Anti-CoL and AFC were similar in both groups ( $p>0.05)$. Further analysis of JIA patients revealed that current age, disease duration, number of active/limited joints, erythrocyte sedimentation rate (ESR), C-reactive protein (CRP), patient/physician visual analog scale (VAS), Juvenile Arthritis Disease Activity Score (JADAS 71), Disease Activity Score 28-Joint Counts (DAS 28), Childhood Health Assessment Questionnaire (CHAQ), Health Assessment Questionnaire (HAQ), patient/parents Pediatric Quality of Life Inventory (PedsQL), physical function-Short-form 36-item questionnaire (SF 36), cumulative glucocorticoid and cumulative methotrexate doses were not correlated with $\mathrm{AMH}, \mathrm{FSH}$, estradiol levels or AFC ( $p>0.05)$. Conclusions: The present study was the first to suggest diminished ovarian reserve, not associated to hypothalamic pituitary gonadal axis, in JIA patients during reproductive age. The impact of this dysfunction in future fertility of these patients needs to be evaluated in prospective studies.

Descriptors: Arthritis, juvenile idiopathic; Ovarian reserve; Anti-Müllerian hormone; Fertility; Anti-corpus luteum antibodies; Menarche. 


\section{INTRODUÇÃO}

A artrite idiopática juvenil (AIJ) é a doença reumática mais prevalente na população pediátrica e é caracterizada por um grupo heterogêneo de doenças, que inclui artrite crônica de etiologia desconhecida e persistente por, no mínimo, 6 semanas e com idade de início inferior a 16 anos $^{1}$. Apresenta uma prevalência estimada em 1 em cada 1.000 crianças $^{2}$.

Atualmente, a AlJ é definida em 7 categorias distintas pela Liga Internacional das Associações de Reumatologia (ILAR): forma sistêmica, poliarticular com fator reumatóide positivo, poliarticular com fator reumatóide negativo, oligoarticular (tendo esta forma duas subdivisões de acordo com a evolução do comprometimento articular após seis meses de doença: persistente ou estendida), artrite psoriásica, artrite associada a entesite e forma indiferenciada ${ }^{3}$.

O curso da doença alterna períodos de atividade e remissão, e a morbidade associada ao diagnóstico tardio inclui, além de questões relacionadas a distúrbio do crescimento e deformidade articular, em estresse psicológico e pode refletir na qualidade de vida dos pacientes ${ }^{1,4,5}$.

Portanto, o tratamento da AlJ visa prevenir e amenizar a injúria articular, reduzir a incapacidade funcional e a dor. E, assim como em outras doenças reumáticas crônicas, o tratamento é realizado através da indução da remissão permanente com a mínima toxicidade possível ${ }^{6}$. Os medicamentos mais utilizados incluem anti-inflamatórios não-hormonais, corticosteroides sistêmicos 
e intra-articulares, drogas antirreumáticas modificadoras de doença (DMARDs) não biológicos, como metotrexato, e agentes biológicos².

Essa doença inflamatória crônica ocorre em adolescentes, jovens e mulheres adultas, principalmente durante a idade reprodutiva e, portanto, a reserva ovariana e a fertilidade futura são relevantes.

A avaliação completa da função gonadal feminina em doenças reumáticas juvenis inclui novos marcadores de reserva ovariana como os níveis do hormônio anti-Mülleriano (HAM) e ultrassom ovariano ${ }^{7,8,9,10}$ e anticorpo anti-corpo lúteo (anti-CoL) $^{10,11}$.Entretanto, esses aspectos foram apenas estudados em pacientes com lúpus eritematoso sistêmico juvenil (LESJ) e dermatomiosite juvenil (DMJ) ${ }^{11,12,13}$.

O conhecimento da dinâmica gonadal feminina é importante para compreensão dos marcadores de reserva ovariana. O ciclo menstrual representa essas modificações cíclicas, sendo dividido em duas fases, a folicular e a lútea, e ocorre sob regulação do eixo hipotálamo-hipófise-gônada, cursando com modificações uterinas secundariamente ${ }^{14,15}$.

O primeiro dia do ciclo, início da fase folicular, inicia-se no primeiro dia da menstruação e cursa com níveis aumentados de hormônio folículo estimulante (FSH), gonadotrofina responsável pela fase de recrutamento de folículos secundários ${ }^{15}$. Durante um único ciclo menstrual, aproximadamente 1.000 folículos são recrutados. No entanto, somente um folículo alcança todas as etapas, sendo caracterizado como folículo dominante ${ }^{7}$.

O aumento do estradiol produzido pelos folículos em crescimento desencadeia as alterações proliferativas no endométrio e estimula o aumento de ambas as gonadotrofinas, principalmente do hormônio luteinizante (LH). 
Aproximadamente no $14^{\circ}$ dia do ciclo, ocorre o pico de $\mathrm{LH}$, que culmina na ovulação e, dá-se início, a fase lútea com a formação do corpo lúteo. Este produzirá estrogênio e progesterona, responsáveis pelas alterações secretórias do endométrio. Na ausência de fecundação, o corpo lúteo sofre atresia e ocorre a descamação do endométrio por declínio do aporte hormonal, portanto, havendo nova menstruação e, reiniciando-se o ciclo ${ }^{14,15}$.

O HAM é secretado pelas células da granulosa dos folículos ovarianos em crescimento e pode ser usado como marcador sérico fidedigno de reserva ovariana $^{10,11,12,13}$, pois estima quantidade e atividade das unidades recrutáveis de uma coorte inicial de folículos em estágios precoces de maturação. Além disto, tem a vantagem de se manter relativamente estável durante todo o ciclo menstrual ${ }^{10}$.

A contagem de folículos antrais (CFA), realizada através do ultrassom ovariano, também é um marcador confiável para a estimativa da reserva ovariana, com correlação positiva com a concentração sérica do HAM ${ }^{14,16}$.

A presença do anticorpo anti-corpo lúteo (anti-CoL) tem aspecto relevante na disfunção gonadal das doenças autoimunes. Este foi evidenciado em $22 \%$ de pacientes com LES ${ }^{17}$, sendo associado a irregularidades menstruais e níveis elevados de FSH, sugerindo ser um marcador precoce de disfunção ovariana, antecedendo a insuficiência ovariana prematura.

Assim sendo, a ausência de estudos avaliando a reserva ovariana em pacientes com AlJ e sua possível associação com dados demográficos, medicamentos, parâmetros de atividade da doença e presença do anti-CoL estimulou a realização desta pesquisa. 


\section{HIPÓTESES}

As hipóteses do presente estudo são que pacientes com AlJ apresentam redução da reserva ovariana em relação aos controles saudáveis. Além disto, a redução da reserva ovariana pode estar relacionada a atividade da doença, presença do anti-CoL e utilização dos medicamentos, particularmente metotrexate ou agentes biológicos. 


\section{OBJETIVOS}

1- Avaliar a reserva ovariana e anti-CoL em pacientes com AIJ e controles saudáveis.

2- Verificar possível associação entre reserva ovariana e: dados demográficos, história ginecológica, parâmetros clínicos e laboratoriais da atividade da doença, presença do anti-CoL e tratamento. 


\section{MÉTODOS}

\subsection{Desenho do estudo e população}

Um estudo transversal foi realizado de setembro de 2014 a junho de 2016 e 110 pacientes com AlJ do sexo feminino, com idades entre 15 a 40 anos, foram convidadas. Todas as pacientes preencheram os critérios da Liga Internacional Contra o Reumatismo (ILAR) 3 para o diagnóstico de AlJ e foram provenientes de dois serviços terciários de Reumatologia: Unidade de Reumatologia Pediátrica do Instituto da Criança e do Adolescente do Hospital das Clínicas da Faculdade de Medicina da Universidade de São Paulo (HC-FMUSP) e Disciplina de Reumatologia do HC-FMUSP.

Os critérios de exclusão foram: contraindicação ou recusa em suspender o uso de contraceptivos hormonais por pelo menos 6 meses ou até retorno da menstruação por 3 meses consecutivos ou recusa em participar desse estudo $(n=64)$, amenorreia $(n=3)$, tireoidopatias $(n=6)$, gestação ou lactação atual $(n=3)$, síndrome do ovário policístico $(n=2)$, presença de outra doença sistêmica autoimune $(n=2)$, obesidade em pacientes virgens, pela dificuldade em se realizar a ultrassonografia abdominal $(n=1)$ e disfunção do eixo hipotálamohipófise-gônada $(n=1)$. Nenhuma delas tinha história de cirurgia ginecológica, irradiação pélvica devido a neoplasia, uso de análogo de hormônio liberador de gonadotrofina, doença renal terminal ou insuficiência ovariana prematura, definida por amenorreia mantida por tempo superior a um ano, idade inferior a 40 anos, aumento nos níveis do FSH acima de 40 UI/L e diminuição nos níveis de estrógeno ${ }^{10}$. 
Assim sendo, após a exclusão de 82 pacientes, 28 pacientes AlJ póspúberes foram estudadas. O grupo controle saudável, pareado por idade, incluiu 28 adolescentes sexo feminino pós-púberes e adultas jovens, todas entre 15 e 40 anos de idade, selecionadas consecutivamente usando os mesmos critérios de exclusão. Este grupo foi procedente de familiares ou conhecidas das pacientes com AlJ. O Comitê de Ética local aprovou o estudo e um consentimento informado foi obtido de todas as participantes e de seus responsáveis legais.

\subsection{Dados demográficos}

Os dados demográficos incluíram: idade atual, raça, classificação socioeconômica ${ }^{18}$, duração da doença e índice de massa corporal (IMC). IMC foi definido como o peso em quilogramas dividido pelo quadrado da altura $\left(\mathrm{m}^{2}\right)$.

\subsection{Avaliação ginecológica}

A idade da menarca em pacientes com AlJ e controles foi registrada. A duração da menstruação e o intervalo do ciclo foram avaliados prospectivamente por, pelo menos, 3 meses consecutivos. Os ciclos normais foram definidos como a duração da menstruação variando de 3 a 7 dias e o intervalo do ciclo de 25 a 35 dias $^{19,20}$. Distúrbios menstruais foram baseados em alterações em um ou mais desses parâmetros durante a avaliação. A média da duração do intervalo do ciclo e a duração da menstruação também foram avaliadas. A amenorreia foi definida como a interrupção de menstruação por três ciclos consecutivos ou um período

maior que seis meses em mulheres que menstruavam previamente ${ }^{19,20}$. A 
presença de dismenorreia, menorragia, síndrome pré-menstrual, presença de muco cervical e dor no meio do ciclo também foram sistematicamente avaliadas.

\subsection{Parâmetros da reserva ovariana}

A função ovariana e a determinação dos níveis séricos hormonais foram avaliadas na fase folicular precoce, nos primeiros cinco dias do ciclo menstrual para ambos os grupos. Os hormônios foram coletados através de punção venosa periférica, pela manhã, entre 8:00 e 9:00 horas. Esses hormônios foram avaliados "cegamente" em relação aos outros parâmetros da função ovariana, ou seja, sem conhecimento prévio do resultado da avaliação ultrassonográfica. $\mathrm{FSH}$, LH, estradiol, testosterona total matutina, progesterona e prolactina foram mensurados por eletroquimioluminescência usando um kit comercial (Roche Diagnostics, Cobas 6000 analyzer series, Mannheim, Germany). Os coeficientes de variação intra e inter-ensaio foram recomendados pelo fabricante e limitados a $2,2-5,1 \%$ e $1,9-8,4 \%$, respectivamente. HAM foi medido por ensaio de quimioluminescência (AMH, Beckman Coulter,UniCel Dxi 800, Brea, CA) e os coeficientes de variação intra e inter-ensaio foram limitados a 9,0 e 7,8\%, respectivamente. Exames de ultrassonografia foram realizados por observador único (RBT), especialista em reprodução humana, em ambos os grupos, com transdutor endovaginal de 4-10 MHz ou abdominal de 2-8 MHz (Voluson S6, GE Healthcare Ultrasound). A CFA e a medição do volume ovariano foram realizadas através de ultrassonografia transvaginal (nas pacientes AlJ e nas pacientes do grupo controle e sexualmente ativas) ou abdominal (nas pacientes AlJ e nas pacientes do grupo controle e virgens) na Disciplina de Ginecologia da HCFMUSP, no mesmo dia da coleta sanguínea. Portanto, a contagem do número 
de folículos antrais foi realizada na fase folicular inicial, levando-se em consideração o número de folículos entre 2 e $10 \mathrm{~mm}^{21}$. Os ovários foram medidos em três planos perpendiculares e o volume ovariano foi calculado através da fórmula da elipsóide (volume = D1 x D2 x D3 x 0,523), sendo D1, D2 e D3 os três diâmetros máximos nos planos longitudinal, ântero-posterior e transverso, respectivamente ${ }^{22}$.

A reserva ovariana diminuída (ROD) foi caracterizada por níveis de HAM $<1,0 \mathrm{ng} / \mathrm{mL}$ ou CFA $<7^{23}$ em ambos os grupos.

\subsection{Detecção do anti-CoL}

A reatividade sérica para a proteína de 67 kDa do corpo lúteo foi avaliada por "immunoblotting" em amostras duplicadas de pacientes com AlJ e controles saudáveis, como descrito anteriormente ${ }^{17}$. Os extratos de células e tecidos de corpo lúteo bovino foram submetidos à eletroforese em gel de poliacrilamida (SDS-PAGE) na presença de sulfato dissódico usando o gel de poliacrilamida a 12\%. Procedeu-se então à transferência eletroforética dos extratos a uma membrana de nitrocelulose cortada em tiras, as quais foram incubadas em solução bloqueadora de leite desnatado a 5\% em tampão fosfato salino (PBS) por duas horas a temperatura ambiente sob agitação contínua. As tiras foram então incubadas com soro diluído (1:10) em PBS contendo 15\% de soro bovino adulto inativado, 3\% de leite desnatado e 0,05\% de polissorbato 20. Após a lavagem, as tiras foram incubadas com $\lg G$ de cabra anti-lgG humana misturada com fosfatase alcalina. A reação foi visualizada adicionando-se os substratos cromogênicos fosfato 5-bromo-4-cloro-3-indolil (BCIP) e nitroazul de tetrazólio ${ }^{17}$. 


\subsection{Parâmetros da doença, qualidade de vida relacionada à saúde e tratamento}

A avaliação clínica das pacientes com AlJ incluiu o número de articulações ativas e limitadas e a avaliação global de atividade da AlJ pelo paciente e pelo médico, por meio de uma escala visual analógica (EVA) horizontal de $100 \mathrm{~mm}$. A atividade da doença AlJ foi avaliada pelos instrumentos "Juvenile Arthritis Disease Activity Score" (JADAS71) (intervalo: 0-101 pontos) para pacientes até $18 \operatorname{anos}^{24}$ e "Disease Activity Score 28-Joint Counts" (DAS28) para pacientes acima de 18 anos $^{25}$. A versão brasileira validada do "Childhood Health Assessment Questionnaire" (CHAQ) ${ }^{26}$ foi usada para pacientes AlJ com menos de 18 anos de idade e "Health Assessment Questionnaire" (HAQ) para pacientes AlJ maiores de 18 anos $^{27}$. A qualidade de vida relacionada à saúde foi avaliada com a versão brasileira "Pediatric Quality of Life Inventory" (PedsQL) para pacientes AlJ com até 18 anos $^{28}$ e questionário "Short-form 36-item questionnaire" (SF-36) para pacientes AlJ com mais de 18 anos ${ }^{29}$. Avaliações laboratoriais incluíram proteína C-reativa (PCR) determinada por nefelometria e velocidade de hemossedimentação (VHS) avaliada pelo método Westergren.

Uso de anti-inflamatórios não esteroidais, corticosteroides, drogas antirreumáticas modificadoras da doença - DMARDs (metotrexato, leflunomida, sulfassalazina e hidroxicloroquina), ciclofosfamida e agentes biológicos (etanercepte, adalimumabe, abatacepte e tocilizumabe) foram sistematicamente avaliados através dos prontuários, considerando a dosagem de uso atual e cumulativa. 


\section{ANÁLISE ESTATÍSTICA}

Baseado na amostra de 25 pacientes com AlJ e considerando a informação da literatura (36\% de disfunção ovulatória/redução da reserva ovariana versus $3 \%$ nos controles), a casuística de 25 casos com AlJ conferiu ao estudo $80 \%$ de poder de encontrar diferenças iguais ou superiores a 26\% (software GraphPad StatMate).

Os resultados para as variáveis contínuas foram apresentados por mediana (valor mínimo e valor máximo) ou média \pm desvio padrão (DP), e para variáveis categóricas apresentadas em frequência (porcentagem). Os escores que tiveram distribuição normal e anormal foram comparados pelo teste-t de Student e pelo teste de Mann-Whitney, respectivamente. As diferenças das variáveis categóricas foram calculadas pelo teste exato de Fisher ou pelo teste quiquadrado de Pearson, conforme apropriado. Os coeficientes de correlação de Pearson ou Spearman foram usados para correlações entre os parâmetros de reserva ovariana e as variáveis demográficas, da doença e do tratamento. 0 nível de significância adotado em todas as análises foi estabelecido em $5 \%$. 


\section{RESULTADOS}

De acordo com o subtipo da doença, a AlJ de início oligoarticular foi observada em $3 / 28$ pacientes (11\%), início poliarticular em 19/28 (68\%), início sistêmico em 5/28 (18\%) e artrite relacionada à entesite em 1/28 (3\%). A reserva ovariana diminuída foi semelhante em pacientes com AlJ e controles saudáveis $(11 \%$ vs. $11 \%, p=1,000)$.

A Tabela 1 mostra dados demográficos e avaliação ginecológica de pacientes com AlJ e controles saudáveis. A média de idade atual foi semelhante em pacientes com AlJ e controles saudáveis ( $22,60 \pm 6,59$ vs. $22,50 \pm 6,59$ anos, $\mathrm{p}=0,952$ ). Entretanto, a mediana de idade da menarca foi significantemente maior em pacientes com AlJ quando comparada ao grupo controle [13 (8-16) vs. 12 (8-14) anos, $p=0,029]$. Não foram observadas diferenças nos parâmetros dos ciclos menstruais em ambos os grupos $(p>0,05)$.

A Tabela 2 apresenta os níveis séricos hormonais e outros parâmetros ovarianos e anti-CoL em pacientes com AlJ e controles saudáveis. Uma mediana significantemente menor dos níveis de HAM foi observada em pacientes com AlJ em comparação com controles $[2,65(0,47-9,08)$ vs. $4,83(0,74-17,24) \mathrm{ng} / \mathrm{mL}$, $\mathrm{p}=0,029]$. Maiores níveis séricos de LH $(8,44 \pm 4,14$ vs. $6,03 \pm 2,80 \mathrm{UI} / \mathrm{L}, \mathrm{p}=0,014)$ e de estradiol $[52,3(25,8-227,4)$ vs. $38,9(26,2-133,6) \mathrm{pg} / \mathrm{mL}, \mathrm{p}=0,008]$ foram significantemente observados em AlJ versus controles. As frequências de antiCoL ( $3 \%$ vs. $3 \%, p=1,0)$ e mediana da CFA [16,5 (6-57) vs. $20(2-37), p=0,222]$ foram semelhantes nos dois grupos $(p>0,05)$. 
Ciclofosfamida endovenosa foi utilizada em apenas duas pacientes, com uma dose cumulativa total inferior a 4 gramas. Ambas apresentavam parâmetros ovarianos normais.

Quanto às correlações de Pearson ou Spearman para pacientes com AlJ, a idade atual, duração da doença, número de articulações ativas e limitadas, VHS, PCR, EVA do paciente e do médico, JADAS 71, DAS 28, CHAQ, HAQ, PedsQL pelo paciente e pelos pais, função física do SF 36, doses cumulativas de corticosteroide e metotrexato não foram correlacionadas com os níveis de HAM, FSH, estradiol ou CFA ( $p>0,05)$.

Tabela 1 - Dados demográficos e avaliação ginecológica em pacientes com artrite idiopática juvenil (AIJ) e controles saudáveis

\begin{tabular}{lccc}
\hline Variáveis & $\begin{array}{c}\text { AlJ } \\
(\mathbf{n = 2 8})\end{array}$ & $\begin{array}{c}\text { Controles } \\
\text { saudáveis } \\
(\mathbf{n = 2 8})\end{array}$ & $\boldsymbol{P}$ \\
\hline Dados demográficos & & & \\
Idade atual, anos & $22,6 \pm 6,59$ & $22,5 \pm 6,59$ & 0,952 \\
Caucasianos & $16(57)$ & $20(71)$ & 0,403 \\
Classes socioeconômicas B ou C & $27(96)$ & $22(79)$ & 0,101 \\
Índice de massa corpórea, kg/m² & $22,1(17,4-29,9)$ & $21,8(19,2-35,8)$ & 0,991 \\
Avaliação ginecológica & & & \\
Ciclo Menstrual & $13(8-16)$ & $12(8-14)$ & 0,029 \\
Idade da menarca, anos & $5(3-8)$ & $5(3-9)$ & 0,451 \\
Duração do fluxo menstrual, dias & $28(24-42)$ & $28(15-42)$ & 0,787 \\
Duração do ciclo, dias & $16(57)$ & $11(39,3)$ & 0,285 \\
Dismenorreia & $3(11)$ & $4(14)$ & 1,000 \\
Menorragia & $21(75)$ & $15(54)$ & 0,162 \\
Síndrome pré-menstrual & $19(68)$ & $17(61)$ & 0,585 \\
Presença de muco cervical & $11(39)$ & $11(39)$ & 1,000 \\
Dor no meio do ciclo & &
\end{tabular}


Tabela 2 - Parâmetros hormonais, parâmetros ovarianos e anticorpo anti-corpo lúteo em pacientes com artrite idiopática juvenil (AIJ) e controles

saudáveis

\begin{tabular}{|c|c|c|c|}
\hline Variáveis & $\begin{array}{c}\text { AlJ } \\
(n=28)\end{array}$ & $\begin{array}{c}\text { Controles } \\
\text { saudáveis } \\
(\mathrm{n}=28)\end{array}$ & $\boldsymbol{P}$ \\
\hline $\begin{array}{l}\text { Parâmetros hormonais } \\
\text { ovarianos }\end{array}$ & & & \\
\hline $\mathrm{FSH}, \mathrm{IU} / \mathrm{L}$ & $6,4(3,7-12,2)$ & $6,3(2,6-12,7)$ & 0,317 \\
\hline $\mathrm{LH}, \mathrm{IU} / \mathrm{L}$ & & 2,80 & 0,014 \\
\hline Valores elevados & & & 0,611 \\
\hline Estradiol, $\mathrm{pg} / \mathrm{mL}$ & $52,3(25,8-227,4)$ & $38,9(26,2-133,6)$ & 0,008 \\
\hline vados, $>233 \mathrm{pg} / \mathrm{mL}$ & & $0(0)$ & 1,000 \\
\hline HAM, $r$ & $2,65(0$, & $4,83(0,74-17,24)$ & 0,029 \\
\hline Valores reduzido, $<1,0 \mathrm{ng} /$ & $2(7)$ & $2(7)$ & 1,000 \\
\hline ariano direito, $\mathrm{cm}^{3}$ & $4,9(1$, & $6,1(2-16,1)$ & 0,099 \\
\hline Volume ovariano esquerdo, $\mathrm{cm}^{3}$ & 5,1 (2) & $5,6(2,1-15,6)$ & 0,966 \\
\hline CFA & 16 , & 20( & 0,222 \\
\hline ido, $<7$ & & $1(3)$ & 1,000 \\
\hline Progesterona, $\mathrm{ng} / \mathrm{mL}$ & $0,4(0,2-1,2)$ & $0,4(0-1,5)$ & 0,935 \\
\hline Testosterona total matutina, $\mathrm{ng} / \mathrm{dL}$ & $26(11-90)$ & $19,5(11-68)$ & 0,317 \\
\hline Prolactina, $\mathrm{ng} / \mathrm{mL}$ & $13,3(5,6-29)$ & $11(4,4-31)$ & 0,108 \\
\hline & & $3(11)$ & \\
\hline Anticorpo anti-corpo lúteo & $1(3)$ & $1(3)$ & 1,000 \\
\hline
\end{tabular}

Valores expressos como $\mathrm{n}(\%)$, mediana (intervalo) ou média \pm desvio padrão, FSH - hormônio folículo estimulante, LH - hormônio luteinizante, HAM hormônio anti-Mülleriano, CFA - contagem de folículo antral.

Não foram observadas diferenças entre as pacientes com AlJ que receberam dose cumulativa de metotrexato $\geq 5 \mathrm{~g}(\mathrm{n}=14)$ em comparação àquelas que receberam dose cumulativa de metotrexato $<5 \mathrm{~g}(\mathrm{n}=12)$ em relação aos parâmetros ovarianos: $\mathrm{FSH}(6,89 \pm 1,21$ vs. $7,20 \pm 2,30 \mathrm{UI} / \mathrm{L}, \mathrm{p}=0,674)$, LH [6,9 $(2,4-13,2)$ vs. $8,3(4,1-23,2) \mathrm{UI} / \mathrm{L}, \mathrm{p}=0,777]$, estradiol $[54(35,5-160,2)$ vs. 52,5 $(25,8-227,4) \mathrm{pg} / \mathrm{mL}, \mathrm{p}=0,857]$, HAM $[2,24(0,47-8,85)$ vs. $3,01(1,03-9,08) \mathrm{ng} / \mathrm{mL}$, $p=0,554]$, volume ovariano direito $\left(4,39 \pm 1,66\right.$ vs. $\left.6,17 \pm 2,88 \mathrm{~cm}^{3}, p=0,071\right)$, volume ovariano esquerdo $\left[5,8(2,2-27,1)\right.$ vs. $\left.5,4(2,7-8) \mathrm{cm}^{3}, \mathrm{p}=0,662\right]$ e CFA [13,5 (8-40) vs. 17,5 (6-57), $p=0,247]$ (Tabela 3). 
Não foram evidenciadas diferenças entre pacientes com AlJ que receberam agentes biológicos $(n=17)$ comparados àqueles que não receberam esses medicamentos $(n=11)$ em relação a todos os parâmetros ovarianos avaliados: FSH $(6,91 \pm 1,89$ vs. $7,04 \pm 1,78 \mathrm{IU} / \mathrm{L}, \mathrm{p}=0,860)$, LH $[7,8(4,2-23,2)$ vs. $7,9(2,4-$ 16) $\mathrm{Ul} / \mathrm{L}, \mathrm{p}=0,778]$ estradiol $[47,4(25,8-227,4)$ vs. $57,1(32,7-160,2) \mathrm{pg} / \mathrm{mL}$, $\mathrm{p}=0,672]$, níveis de HAM $[3,36(0,64-9,08)$ vs. $2,42(0,47-6,47) \mathrm{ng} / \mathrm{mL}, \mathrm{p}=0,323]$, volume ovariano direito $\left(5,47 \pm 2,31\right.$ vs. $\left.5,07 \pm 2,72 \mathrm{~cm}^{3}, \mathrm{p}=0,677\right)$, volume ovariano esquerdo $\left[6,3(2,5-9,3)\right.$ vs. $\left.4,2(2,2-27,1) \mathrm{cm}^{3}, \mathrm{p}=0,100\right]$ e CFA [16 (657) vs. 18 (8-34), $p=0,906$ ] (Tabela 4).

Tabela 3 - Avaliação hormonal em pacientes com artrite idiopática juvenil (AIJ) que utilizaram metotrexato (MTX) acima da dose cumulativa de 5 gramas versus aqueles que usaram dose cumulativa abaixo de 5 gramas

\begin{tabular}{|c|c|c|}
\hline Variáveis & $\begin{array}{c}M T X \geq 5 \\
\operatorname{gramas} \\
(n=14)\end{array}$ & $\begin{array}{c}\text { MTX }<5 \\
\text { gramas } \\
(n=12)\end{array}$ \\
\hline
\end{tabular}

\begin{tabular}{lccc}
\hline $\begin{array}{l}\text { Reserva ovariana e outros } \\
\text { hormônios }\end{array}$ & & & \\
FSH, UI/L & $6,89 \pm 1,21$ & $7,20 \pm 2,30$ & 0,674 \\
$\mathrm{LH}, \mathrm{UI} / \mathrm{L}$ & $6,9(2,4-13,2)$ & $8,3(4,1-23,2)$ & 0,777 \\
Estradiol, pg/mL & $54(35,5-160,2)$ & $52,5(25,8-227,4)$ & 0,857 \\
$\mathrm{HAM}, \mathrm{ng} / \mathrm{mL}$ & $2,24(0,47-8,85)$ & $3,01(1,03-9,08)$ & 0,554 \\
Volume ovariano direito, $\mathrm{cm}^{3}$ & $4,39 \pm 1,66$ & $6,17 \pm 2,88$ & 0,071 \\
Volume ovariano esquerdo, $\mathrm{cm}^{3}$ & $5,8(2,2-27,1)$ & $5,4(2,7-8)$ & 0,662 \\
CFA & $13,5(8-40)$ & $17,5(6-57)$ & 0,247 \\
\hline
\end{tabular}

Valores expressos em $\mathrm{n}$ (\%) ou mediana (intervalo), FSH - hormônio folículo estimulante, LH - hormônio luteinizante, HAM - hormônio anti-Mülleriano, CFA - contagem de folículos antrais. 
Tabela 4 - Avaliação hormonal em pacientes com artrite idiopática juvenil (AlJ) que utilizaram ou não agentes biológicos.

\begin{tabular}{lccc}
\hline Variáveis & $\begin{array}{c}\text { Usaram } \\
\text { agentes } \\
\text { biológicos } \\
(\mathbf{n = 1 7 )}\end{array}$ & $\begin{array}{c}\text { Não usaram } \\
\text { agentes } \\
\text { biológicos } \\
(\mathbf{n = 1 1 )}\end{array}$ & $\boldsymbol{p}$ \\
\hline Reserva ovariana & $6,91 \pm 1,89$ & $7,04 \pm 1,78$ & 0,860 \\
$\mathrm{FSH}, \mathrm{UI} / \mathrm{L}$ & $7,8(4,2-23,2)$ & $7,9(2,4-16)$ & 0,778 \\
$\mathrm{LH}, \mathrm{UI} / \mathrm{L}$ & $47,4(25,8-227,4)$ & $57,1(32,7-160,2)$ & 0,672 \\
Estradiol, pg/mL & $3,36(0,64-9,08)$ & $2,42(0,47-6,47)$ & 0,323 \\
HAM, ng/mL & $5,47 \pm 2,31$ & $5,07 \pm 2,72$ & 0,677 \\
Volume ovariano direito, $\mathrm{cm}^{3}$ & $6,3(2,5-9,3)$ & $4,2(2,2-27,1)$ & 0,100 \\
Volume ovariano esquerdo, $\mathrm{cm}^{3}$ & $16(6-57)$ & $18(8-34)$ & 0,906 \\
CFA & & & \\
\hline
\end{tabular}

Valores expressos em $n(\%)$, mediana (intervalo) ou média \pm desvio padrão, FSH - hormônio folículo estimulante, LH - hormônio luteinizante, HAM - hormônio anti-Mülleriano, CFA - contagem de folículos antrais.

Não foram evidenciadas diferenças entre as pacientes cujo início da AlJ ocorreu após a menarca $(n=9)$ em comparação com início da AlJ antes da menarca $(n=19)$ em relação aos parâmetros ovarianos: FSH [6,2 (3,7-10,1) vs. $6,4(4,7-12,2) U I / L, p=0,786)$, LH $[6,5(2,4-13,2)$ vs. $7,9(4,2-23,2) U I / L, p=0,460]$, estradiol $[41,5(25,8-227,4)$ vs. 53,5 $(37,6-160,2) \mathrm{pg} / \mathrm{mL}, \mathrm{p}=0,089]$, níveis de HAM $[2,65(0,64-6,47)$ vs. $3,36(0,47-9,08) \mathrm{ng} / \mathrm{mL}, \mathrm{p}=0,824]$, volume ovariano direito $\left[4,4(3,2-8,6)\right.$ vs. $\left.5,6(1,5-12,8) \mathrm{cm}^{3}, \mathrm{p}=0,325\right)$, volume ovariano esquerdo $[5,05$ $(2,2-27,1)$ vs. $\left.5,6(2,2-8,9) \mathrm{cm}^{3}, \mathrm{p}=0,948\right]$ e CFA [14 (9-33) vs. 18 (6 -57), $\mathrm{p}=0,475]$ (Tabela 5). 
Tabela 5 - Avaliação hormonal em pacientes com artrite idiopática juvenil (AIJ) cujo início da AlJ ocorreu após a menarca versus início da AlJ antes da menarca

\begin{tabular}{|c|c|c|c|}
\hline Variáveis & $\begin{array}{c}\text { AlJ após } \\
\text { menarca } \\
(n=9)\end{array}$ & $\begin{array}{c}\text { AlJ antes da } \\
\text { menarca } \\
(n=19)\end{array}$ & $\boldsymbol{p}$ \\
\hline \multicolumn{4}{|l|}{$\begin{array}{l}\text { Reserva ovariana e outros } \\
\text { hormônios }\end{array}$} \\
\hline FSH, UI/L & $6,2(3,7-10,1)$ & $6,4(4,7-12,2)$ & 0,786 \\
\hline $\mathrm{LH}, \mathrm{UI} / \mathrm{L}$ & 6,5 & 7,9 & 0,460 \\
\hline Estradiol, $\mathrm{pg} / \mathrm{mL}$ & $41,5(25,8-227,4)$ & $53,5(37,6-160,2)$ & 0,089 \\
\hline $\mathrm{HAM}, \mathrm{ng} / \mathrm{mL}$ & $2,65(0,64-6,47)$ & $3,36(0,47-9,08)$ & 0,824 \\
\hline Volume ovariano direito, $\mathrm{cm}^{3}$ & $4,4(3,2-8,6)$ & $5,6(1,5-12,8)$ & 0,325 \\
\hline Volume ovariano esquerdo, $\mathrm{cm}^{3}$ & $5,05(2,2-27,1)$ & $5,6(2,2-8,9)$ & 0,948 \\
\hline CFA & $14(9-33)$ & $18(6-57)$ & 0,475 \\
\hline
\end{tabular}

Valores expressos em $\mathrm{n}$ (\%) ou mediana (intervalo), $\mathrm{FSH}$ - hormônio folículo estimulante, LH - hormônio luteinizante, HAM - hormônio anti-Mülleriano, CFA - contagem de folículos antrais. 


\section{DISCUSSÃO}

Este estudo foi o primeiro a sugerir uma diminuição da reserva ovariana, não associada ao eixo hipotálamo-hipófise-gônada, em pacientes com AIJ durante a idade reprodutiva.

O ponto forte deste estudo foi a realização de uma avaliação completa da reserva ovariana em pacientes com AlJ pós-púberes e controles saudáveis pareados por idade, na fase folicular precoce do ciclo menstrual, fornecendo assim uma estimativa mais precisa da população folicular e da qualidade do oócito $30,31,32,33,34,35,36$. Além disso, a padronização da coleta de sangue matutina foi importante, uma vez que a produção de $\mathrm{FSH}$ apresenta variação circadiana ${ }^{10}$.

Foram excluídas pacientes e controles saudáveis com amenorreia, e, portanto, nenhuma das participantes apresentou insuficiência ovariana prematura. Os critérios rigorosos de seleção das pacientes com AlJ e do grupo controle saudável, com idade inferior a 40 anos, sem doenças ginecológicas e sem disfunção do eixo hipotálamo-hipófise-gônada foram relevantes, uma vez que essas anormalidades podem reduzir a reserva ovariana ${ }^{8,9,10,11,31,32,33,34,35,36}$.

Também foram avaliados os níveis séricos de hormônios e anti-CoL sem o efeito de quaisquer contraceptivos hormonais por, pelo menos, três meses para evitar possíveis vieses, visto que os níveis de HAM podem ser diminuídos em mulheres usando esses métodos de controle de natalidade ${ }^{16}$. No entanto, uma limitação foi o tamanho pequeno da amostra devido aos critérios restritos de inclusão e exclusão. 
O teste dinâmico para avaliar a reserva ovariana não é necessário para o diagnóstico de reserva ovariana diminuída, uma vez que esta foi definida neste estudo como diminuição da CFA ou diminuição dos níveis séricos de $\mathrm{HAM}^{23}$. Os valores significantemente mais baixos de HAM em pacientes com AlJ sugeriram uma redução da população de folículos ovarianos, apesar dos valores comparáveis da CFA. De fato, a CFA tem baixa sensibilidade e alta especificidade ${ }^{37}$.

Embora o FSH sérico basal e o estradiol sejam usados como marcadores de reserva ovariana, demonstraram ter baixa precisão no diagnóstico da reserva ovariana diminuída, uma vez que a confiabilidade de ambos os hormônios é limitada devido à alta variabilidade do ciclo inter e intramenstrual ${ }^{37}$.

A insuficiência ovariana prematura foi previamente descrita em 3,5\% a 7,5\% das pacientes com AIJ, principalmente em pacientes adultas (média da idade de 35 anos) $)^{38}$ e sob o uso de clorambucil para amiloidose ${ }^{39}$. Nenhuma das pacientes deste estudo com AIJ apresentava essa anormalidade, pois eram mais jovens e, também, nunca foram tratadas com esse agente alquilante. Além disso, amenorreia foi um critério de exclusão.

A reserva ovariana diminuída em doenças reumáticas autoimunes pediátricas e em adultos pode ocorrer devido ao envelhecimento, atividade da doença, autoimunidade e uso de agentes imunossupressores ${ }^{7,8,9,19,20,40,41,42,43}$. A idade semelhante entre pacientes com AlJ pós-púberes e controles excluiu essa variável de confusão e corroborou a ideia de reserva ovariana precocemente reduzida em pacientes com AIJ. A atividade da doença não pareceu afetar a saúde reprodutiva das pacientes com AIJ deste estudo, contrastando com a 
doença de Behçet, na qual a exacerbação da doença foi o fator contribuinte mais importante para a redução da reserva ovariana ${ }^{32}$.

A reserva ovariana diminuída em pacientes com AlJ pode ser desencadeada por mecanismos autoimunes. A ooforite autoimune pode afetar os folículos em desenvolvimento, poupando os folículos primordiais durante a fase inicial, devido à ativação das células T CD4 +, induzindo a diminuição progressiva da função ovariana em camundongos. Em humanos, existem vários antígenos alvo relatados envolvidos na ooforite autoimune, como zona pelúcida, oócito, células da granulosa, células da teca, enzimas esteroidogênicas e corpo lúteo ${ }^{10}$. A presença de anti-CoL foi raramente observada nas pacientes com AIJ neste estudo, contrastando com uma prevalência de $16-22 \%$ em adultos e pacientes com LESJ ${ }^{11,17}$.

O metotrexato parece não influenciar a população de folículos ovarianos na AlJ, contrastando com um estudo anterior que demonstrou que uma alta dose cumulativa de metotrexato foi uma possível causa de disfunção ovariana em pacientes adultas com LESJ ${ }^{11}$.

Além disso, os agentes biológicos não parecem comprometer a fertilidade em pacientes do sexo feminino ${ }^{8,9}$, como também observado neste estudo.

Em relação aos agentes imunossupressores, a ciclofosfamida endovenosa não foi um fator contribuinte para a diminuição da reserva ovariana em pacientes com AlJ, diferindo de relatos em pacientes com LESJ ${ }^{11,13}$ e com $\mathrm{DMJ}^{12}$. Este fármaco foi utilizado apenas em duas pacientes, com uma dose cumulativa total inferior a $4 \mathrm{~g}$ e ambas apresentavam parâmetros ovarianos normais.

O atraso na menarca foi observado em pacientes com AlJ versus controles, como também observado em pacientes com LESJ ${ }^{13,19,20,43}$ e DMJ'2 
A menarca representa um evento tardio da puberdade, sendo a telarca o primeiro marco puberal no sexo feminino, precedendo a menarca em aproximadamente 2 anos. Um estudo recente brasileiro demonstrou que a menarca ocorreu com média de idade de 11,7 anos ${ }^{44}$. Diversos fatores parecem influenciar a idade da menarca, como doenças crônicas, uso de medicações como os corticosteroides, estado nutricional, além de fatores genéticos ${ }^{44-48}$. 0 atraso puberal pode ocorrer principalmente quando a doença crônica se inicia no período pré-puberal ${ }^{45,49} \mathrm{e}$, de fato, a maioria das pacientes do presente estudo com AlJ tiveram o início da doença antes da menarca (19/28) e mais de $80 \%$ das pacientes AIJ apresentaram os subtipos poliarticular (68\%) ou sistêmico (18\%), sugerindo doença com maior componente inflamatório. As duas pacientes com AlJ e menarca tardia (aos 16 anos) começaram a doença aos 5 e 6 anos, subtipo poliarticular e sistêmico, respectivamente. Além disso, fizeram uso prolongado de corticoterapia. Ambas as condições podem influenciar os marcos puberais na AlJ 45,48 .

A qualidade de vida relacionada à saúde em adolescentes e mulheres jovens com AlJ não foi estudada previamente em conjunto com parâmetros de reserva ovariana. A despeito da não correlação entre os parâmetros de atividade da doença e qualidade de vida relacionada à saúde com níveis de HAM, FSH, estradiol ou CFA, mais estudos devem ser realizados, visto que, o estresse e a qualidade de vida relacionada à saúde, incluindo fatores psicossociais e econômicos, parecem interferir na fertilidade ${ }^{50}$.

Assim sendo, pacientes com AlJ sugerem ter reserva ovariana diminuída. O impacto dessa disfunção na fertilidade futura dessas pacientes precisa ser avaliado em estudos prospectivos e com maior casuística. 


\section{CONCLUSÕES}

1- Pacientes com AlJ sugerem ter reserva ovariana diminuída e raramente antiCoL.

2- Reserva ovariana diminuída não esteve associada com atividade da doença, presença do anti-CoL e tratamento. 


\section{REFERÊNCIAS}

[1] Prakken B, Albani S, Martini A. Juvenile idiopathic arthritis. Lancet. 2011;377:2138-49.

[2] 2019 American College of Rheumatology/Arthritis Foundation Guideline for the Treatment of Juvenile Idiopathic Arthritis: Therapeutic Approaches for Non-Systemic Polyarthritis, Sacroiliitis, and Enthesitis. Arthritis Rheumatol. 2019;71(6):846-863.

[3] Petty RE, Southwood TR, Manners P, Baum J, Glass DN, Goldenberg J, et al. International League of Associations for Rheumatology classification of juvenile idiopathic arthritis: second revision, Edmonton, 2001. J Rheumatol. 2004;31:390-2.

[4] Pugliese C, van der Vinne RT, Campos LM, Guardieiro PR, Saviolli C, Bonfa E. Juvenile idiopathic arthritis activity and function ability: deleterious effects in periodontal disease? Clin Rheumatol. 2016;35:81-91.

[5] Gualano B, Bonfa E, Pereira RM, Silva CA. Physical activity for paediatric rheumatic diseases: standing up against old paradigms. Nat Rev Rheumatol. 2017;13:368-79.

[6] American College of Rheumatology, Subcommittee on Rheumatoid Arthritis Guidelines. Guidelines for the management of rheumatoid arthritis: 2002 Update. Arthritis Rheum 2002;46:328-46.

[7] Silva CA, Brunner HI. Gonadal functioning and preservation of reproductive fitness with juvenile systemic lupus erythematosus. Lupus. 2007;16:593-9. 
[8] Silva CA, Bonfa E, Østensen M. Maintenance of fertility in patients with rheumatic diseases needing antiinflammatory and immunosuppressive drugs. Arthritis Care Res (Hoboken). 2010;62:1682-90.

[9] Ostensen M, Brucato A, Carp H, Chambers C, Dolhain RJ, Doria A, et al. Pregnancy and reproduction in autoimmune rheumatic diseases. Rheumatology (Oxford). 2011;50:657-64.

[10] Silva CA, Yamakami LY, Aikawa NE, Araujo DB, Carvalho JF, Bonfá E. Autoimmune primary ovarian insufficiency. Autoimmun Rev. 2014;13:427-30.

[11]de Araujo DB, Yamakami LY, Aikawa NE, Bonfá E, Viana VS, Pasoto SG, et al. Ovarian reserve in adult patients with childhood-onset lupus: a possible deleterious effect of methotrexate? Scand J Rheumatol. 2014;43:503-11.

[12] Aikawa NE, Sallum AM, Leal MM, Bonfá E, Pereira RM, Silva CA. Menstrual and hormonal alterations in juvenile dermatomyositis. Clin Exp Rheumatol. 2010;28:571-5.

[13]Aikawa NE, Sallum AM, Pereira RM, Suzuki L, Viana VS, Bonfa E, et al. Subclinical impairment of ovarian reserve in juvenile systemic lupus erythematosus after cyclophosphamide therapy. Clin Exp Rheumatol. 2012;30:445-9.

[14]Jamil Z, Fatima SS, Ahmed K, Malik R. Anti-Müllerian Hormone: above and beyond conventional ovarian reserve markers. Dis Markers. 2016; 5246217.

[15]Barbieri RL. The endocrinology of the menstrual cycle. Methods Mol Biol2014;1154:145-69.

[16] Kristensen SL, Ramlau-Hansen CH, Andersen CY, Ernst E, Olsen SF, Bonde JP et al. The association between circulating levels of antimüllerian hormone 
and follicle number, androgens, and menstrual cycle characteristics in young women. Fertil Steril. 2012;97:779-85.

[17]Pasoto SG, Viana VS, Mendonca BB, Yoshinari NH, Bonfa E. Anti-corpus luteum antibody: a novel serological marker for ovarian dysfunction in systemic lupus erythematosus? J Rheumatol. 1999;26:1087-93.

[18]Critério de Classificação Econômica Brasil. ABEP (Associação Brasileira de Empresas de Pesquisa). 2014;1-5.

[19] Silva CA, Leal MM, Leone C, Simone VP, Takiuti AD, Saito Ml et al (2002) Gonadal function in adolescents and young women with juvenile systemic lupus erythematosus. Lupus. 2002;11:419-25.

[20]Medeiros PB, Febronio MV, Bonfa E, Borba EF, Takiuti AD, Silva CA. Menstrual and hormonal alterations in juvenile systemic lupus erythematosus. Lupus. 2009;18:38-43.

[21]Hendriks DJ, Mol BW, Bancsi LF, Te Velde ER, Broekmans FJ. Antral follicle count in the prediction of poor ovarian response and pregnancy after in vitro fertilization: a meta-analysis and comparison with basal follicle-stimulating hormone level. Fertil Steril. 2005;83:291-301.

[22]Hendriks DJ, Kwee J, Mol BW, Te Velde ER, Broekmans FJ. Ultrasonography as a tool for the prediction of outcome in IVF patients: a comparative meta-analysis of ovarian volume and antral follicle count. Fertil Steril. 2007;87:764-75.

[23] Cohen J, Chabbert-Buffet N, Darai E. Diminished ovarian reserve, premature ovarian failure, poor ovarian responder--a plea for universal definitions. $J$ Assist Reprod Genet. 2015;32:1709-12. 
[24] Consolaro A, Ruperto N, Bazso A, Pistorio A, Magni-Manzoni S, Filocamo G, Paediatric Rheumatology International Trials Organisation, et al. Development and validation of a composite disease activity score for juvenile idiopathic arthritis. Arthritis Rheum. 2009;61:658-66.

[25]van Riel PLCM. The development of the disease activity score (DAS) and the disease activity score using 28 joint counts (DAS28). Clin Exp Rheumatol. 2014;32:65-74.

[26] Machado CS, Ruperto N, Silva CH, Ferriani VP, Roscoe I, Campos LM, et al. Paediatric Rheumatology International Trials Organisation. The Brazilian version of the Childhood Health Assessment Questionnaire (CHAQ) and the Child Health Questionnaire (CHQ). Clin Exp Rheumatol. 2001;19:25-9.

[27] Ferraz MB, Oliveira LM, Araujo PM, Atra E, Tugwell P. Crosscultural reliability of the physical ability dimension of the health assessment questionnaire. $\mathrm{J}$ Rheumatol. 1990;17:813-7.

[28]Klatchoian DA, Len CA, Terreri MT, Silva M, Itamoto C, Ciconelli RM, et al. Quality of life of children and adolescents from São Paulo: reliability and validity of the Brazilian version of the Pediatric Quality of Life Inventory version 4.0 Generic Core Scales. J Pediatr (Rio J). 2008;84:308-15.

[29] Ciconelli RM, Ferraz MB, Santos W, Meinão I, Quaresma MR. Tradução para língua portuguesa e validação do questionário genérico de avaliação de qualidade de vida SF-36 (Brasil SF-36). Rev Bras Rheumatol. 1999; 39:3.

[30] Yamakami LY, de Araujo DB, Silva CA, Baracat EC, de Carvalho JF. Severe hemorrhagic corpus luteum complicating anticoagulation in antiphospholipid syndrome. Lupus. 2011;20:523- 6. 
[31]Mont'Alverne AR, Pereira RM, Yamakami LY, Viana VS, Baracat EC, Bonfá E, et al. Reduced ovarian reserve in patients with takayasu arteritis. J Rheumatol. 2014;41:2055-9.

[32] Mont'Alverne AR, Yamakami LY, Gonçalves CR, Baracat EC, Bonfá E, Silva CA. Diminished ovarian reserve in Behçet's disease patients. Clin Rheumatol. 2015;34:179-83.

[33]Yamakami LY, Serafini PC, de Araujo DB, Bonfá E, Leon EP, Baracat EC, et al. Ovarian reserve in women with primary antiphospholipid syndrome. Lupus. 2014;23:862-7.

[34]Yamakami LY, Serafini PC, de Araujo DB, Bonfá E, Leon EP, Baracat EC, et al. Clark CA, Laskin CA. Ovarian reserve in antiphospholipid syndrome: the jury is still out. Lupus 2015; 24: 773. Lupus. 2015;24:1007.

[35] de Souza FH, Shinjo SK, Yamakami LY, Viana VS, Baracat EC, Bonfá E, et al. Reduction of ovarian reserve in adult patients with dermatomyositis. Clin Exp Rheumatol. 2015;33:44-9.

[36] de Souza FH, da Silva CA, Yamakami LY, dos Santos Trindade Viana V, Bonfá E, Shinjo SK. Reduced ovarian reserve in patients with adult polymyositis. Clin Rheumatol. 2015;34:1795-9.

[37]Testing and interpreting measures of ovarian reserve: a committee opinion. Practice Committee of the American Society for Reproductive Medicine. Fertil Steril. 2015;103:e9-e17.

[38]Packham JC, Hall MA. Premature ovarian failure in women with juvenile idiopathic arthritis (JIA). Clin Exp Rheumatol. 2003;21:347-50. 
[39]David J, Vouyiouka O, Ansell BM, Hall A, Woo P. Amyloidosis in juvenile chronic arthritis: a morbidity and mortality study. Clin Exp Rheumatol. $1993 ; 11: 85-90$

[40]Brouwer J, Laven JS, Hazes JM, Schipper I, Dolhain RJ. Levels of serum anti- Müllerian hormone, a marker for ovarian reserve, in women with rheumatoid arthritis. Arthritis Care Res (Hoboken). 2013;65:1534-8.

[41]Henes M, Froeschlin J, Taran FA, Brucker S, Rall KK, Xenitidis T, et al. Ovarian reserve alterations in premenopausal women with chronic inflammatory rheumatic diseases: impact of rheumatoid arthritis, Behçet's disease and spondyloarthritis on anti-Müllerian hormonelevels. Rheumatology (Oxford). 2015;54:1709-12.

[42] Karakus S, Sahin A, Durmaz Y, Aydin H, Yildiz C, Akkar O, et al. Evaluation of ovarian reserve using anti-müllerian hormone and antral follicle count in Sjögren's syndrome: Preliminary study. J Obstet Gynaecol Res. 2017;43:303-7.

[43]Febronio MV, Pereira RM, Bonfa E, Takiuti AD, Pereyra EA, Silva CA. Inflammatory cervicovaginal cytology is associated with disease activity in juvenile systemic lupus erythematosus. Lupus. 2007;16:430-5.

[44]Barros BS, Kuschnir MCMC, Bloch KV, Silva TLN. ERICA: age at menarche and its association with nutritional status. J Pediatr (Rio J) 2019;95(1):106111.

[45] Machado SH, Xavier RM, Lora PS, Gonçalves LMK, Trindade LR, Marostica PJC. Height and sexual maturation in girls with juvenile idiopathic arthritis.J Pediatr (Rio J) 2020;96(1):100-107. 
[46] Umławska W, Krzyzanowska M. Growth and menarche during remission in children with juvenile idiopathic arthritis. Med Wieku Rozwoj 2009;13(3):194200.

[47]Maher SE , Ali FI. Sexual maturation in Egyptian boys and girls with juvenile rheumatoid arthritis. Rheumatol Int 2013;33(8):2123-6.

[48]Rusconi R, Corona F, Grassi A, Carnelli V. Age at menarche in juvenile rheumatoid arthritis. J Pediatr Endocrinol Metab 2003;16 Suppl 2:285-8.

[49] Traggiai, C, Stanhope R. Disorders of pubertal development. Best Pract Res Clin Obstet Gynaecol 2003;17(1):41-56.

[50]Palomba S, Daolio J, Romeo S, Battaglia FA, Marci R, La Sala GB. Lifestyle and fertility: the influence of stress and quality of life on female fertility. Reprod Biol Endocrinol 2018;16(1):113. 